\title{
Superfluid-Mott-insulator transition in the spin-orbit-coupled Bose-Hubbard model
}

\author{
A. T. Bolukbasi and M. Iskin \\ Department of Physics, Koç University, Rumelifeneri Yolu, 34450 Sariyer, Istanbul, Turkey
}

(Received 3 January 2014; published 4 April 2014)

\begin{abstract}
We consider a square optical lattice in two dimensions and study the effects of both the strength and symmetry of spin-orbit coupling and Zeeman field on the ground-state, i.e., Mott-insulator (MI) and superfluid (SF), phases and phase diagram, i.e., MI-SF phase-transition boundary, of the two-component Bose-Hubbard model. In particular, based on a variational Gutzwiller ansatz, our numerical calculations show that the spin-orbit-coupled SF phase is a nonuniform (twisted) one, with its phase (but not the magnitude) of the order parameter modulating from site to site. Fully analytical insights into the numerical results are also given.
\end{abstract}

DOI: 10.1103/PhysRevA.89.043603

PACS number(s): 03.75.Ss, 05.30.Fk, 03.75.Hh

\section{INTRODUCTION}

Ultracold atoms have proved to be exceptional manybody quantum systems, thanks especially to their tunable single-particle potentials and multiparticle interactions. The experimental ability to control the parameters of the atomic Hamiltonian allow one to simulate and study some of the fundamental aspects of condensed-matter systems, including Bose-Einstein condensation (BEC), bosonic superfluidity, quantum magnetism, many-body spin dynamics, BardeenCooper-Schrieffer (BCS) superfluidity, BEC-BCS crossover, etc. [1-3]. In addition, by loading cold atoms into the periodic optical lattice potentials, which are formed by interfering counterpropagating laser beams, it has also been possible to realize Hubbard-type lattice models and study strongly correlated quantum phenomena [1,2]. In particular, bosonic atoms in an optical lattice, whose low-energy dynamics is well captured by the Bose-Hubbard model [4], provide an ideal platform for the observation of Mott-insulator (MI) and superfluid (SF) phases as well as the MI-SF phase transition between the two [1,2].

Meanwhile, recent discoveries of topological insulators [5], topological superconductors [6], and quantum spin-Hall effect [7] have put topological phases of matter in the spotlight. It turns out that the interaction between the quantum particle's spin and its center-of-mass motion (momentum), i.e., spinorbit coupling (SOC), is at the heart of all of these modern condensed-matter phenomena, and creation and manipulation of a similar (if not identical) effect has been an intriguing possibility for the cold-atom community. However, since quantum gases are charge neutral, they do not directly couple to electromagnetic fields, and this prevented SOC studies in atomic systems until the advent of artificial gauge fields [8-10]. By coupling the internal states of atoms to their momentum via Raman dressing of atomic hyperfine states with near-resonant laser beams, it has recently been possible to engineer atomic systems with Abelian gauge fields. For instance, while there are many proposals for implementing atomic gases with various non-Abelian gauge fields that may give rise to Rashba, Dresselhaus, and Weyl SOCs, several experimental groups have so far achieved only a particular form of an Abelian gauge field that may be characterized as an equal Rashba and Dresselhaus (ERD) SOC [11-17]. Note that a very recent proposal to realize SOC in optical lattices does not rely on laser light to couple different spin states [18]. These experiments naturally lead the way to numerous other works on spin-orbit-coupled atomic systems, e.g., topological superfluid phases of matter, bringing once again the condensed-matter and atomic-physics communities together.

Motivated by these developments, here we consider a square lattice in two dimensions and study the effects of both the strength and symmetry of SOC and Zeeman field on the ground-state phases and phase diagram of the two-component Bose-Hubbard model. In particular, based on a variational Gutzwiller ansatz, we analyze the competition between the interaction, tunneling, Rashba and ERD SOCs, and out-ofand in-plane Zeeman fields on the MI-SF phase-transition boundary and the nature of the SF phase nearby. In addition to the phase diagrams, one of our main results is as follows: Gutzwiller calculations show that while the magnitudes of the order parameters are uniform across the entire lattice, their phases may vary from site to site due to SOC, and, therefore, the SF phase is a nonuniform one. We give a complete account and intuitive understanding of this SOC-induced nonuniformSF phase and its resultant phase patterns by supporting our numerical calculations with fully analytical insights.

The rest of the paper is organized as follows. In Sec. II, we introduce the spin-orbit-coupled two-component BoseHubbard model and derive the self-consistency (total average number, polarization, and SF order parameter) equations using a variational Gutzwiller ansatz. Our numerical results for the ground-state MI, uniform-SF and nonuniform-SF phases, and the MI-SF phase-transition boundary are presented in Sec. III as functions of the strength and symmetry of the SOC and Zeeman field. The paper is concluded with a brief summary of our results and an outlook in Sec. IV.

\section{TWO-COMPONENT BOSE-HUBBARD MODEL}

It has long been established that the low-energy dynamics of quantum gases loaded into the periodic optical lattice potentials is well described by Hubbard-type tight-binding lattice models $[1,2]$. In particular, the simplest Bose-Hubbard model [4], which takes into account the chemical potential and nearest-neighbor tunneling of atoms, and short-range (on-site) repulsive interparticle interactions, has proved to be quite successful in describing some of the cold-atom 
experiments where MI and uniform-SF phases as well as the MI-SF phase transition were observed [2]. This success generated an enormous interest in this topic, and many extensions of Hubbard-type models have not only been proposed but also realized in the recent literature, including different lattice geometries, longer-ranged tunnelings and interactions, multiple components, gauge fields, etc. A number of theoretical methods have been developed to tackle these lattice models, and among those the validity and limitations of the variational Gutzwiller ansatz [19], decoupling mean-field theory [20], strong-coupling expansion [21,22], and quantum Monte Carlo [22,23] approaches are well understood.

In this context, the two-component Bose-Hubbard model [24-31] was introduced about a decade ago to describe cold-atom experiments involving two types of bosons, in which the two components may correspond to different hyperfine states of a particular atom or different species of atoms. In addition to the phases that are similar in many ways to the MI and SF phases of the single-component model, these works proposed that paired-SF, counterflow-SF, density-wave insulator, and supersolid phases may be created with the experimental realization of the two-component model. These possibilities already motivated a number of experimental studies on two-component systems [32-35], opening up a new frontier waiting to be explored in the near future.

In addition, excited by the recent realization of spin-orbitcoupled atomic BEC [11,12,18], there has been growing interest in studying the effects of SOC on the two-component model [36-47]. For instance, it has been proposed that the SOC gives rise to rich phase diagrams which exhibit spin textures in the form of spin spirals and vortex and Skyrmion crystals within the MI phase [36-40], and also a nonuniform twisted SF phase [42]. Our main goal here is to provide a complete analysis of this SOC-induced nonuniform-SF phase as well as the MI-SF phase-transition boundary.

\section{A. Hamiltonian: SOC and Zeeman fields}

In this paper, we consider a square lattice in two dimensions and study the effects of both the strength and symmetry of SOC and Zeeman field on the ground-state phases and phase diagram of the two-component Bose-Hubbard model. The Hamiltonian of such systems may be written as

$$
\begin{aligned}
H= & \sum_{j \alpha}\left[\frac{U_{\alpha \alpha}}{2} \hat{n}_{j \alpha}\left(\hat{n}_{j \alpha}-1\right)-\mu_{\alpha} \hat{n}_{j \alpha}\right]+U_{\uparrow \downarrow} \sum_{j} \hat{n}_{j \uparrow} \hat{n}_{j \downarrow} \\
& -\sum_{\langle j, k\rangle}\left(\hat{\Psi}_{j}^{\dagger} T^{j k} \hat{\Psi}_{k}+\text { H.c. }\right)-h_{y} \sum_{j} \hat{\Psi}_{j}^{\dagger} \sigma_{y} \hat{\Psi}_{j},
\end{aligned}
$$

where $\alpha \equiv(\uparrow, \downarrow)$ denotes the two types of bosons, $U_{\alpha \alpha} \geqslant 0$ is the intracomponent and $U_{\uparrow \downarrow} \geqslant 0$ is the intercomponent interaction with $U_{\uparrow \downarrow}^{2}<U_{\uparrow \uparrow} U_{\downarrow \downarrow}$ to prevent phase separation, and the operator $\hat{n}_{j \alpha}=\hat{a}_{j \alpha}^{\dagger} \hat{a}_{j \alpha}$ counts the local number of $\alpha$ bosons at site $j$. Here, the operator $\hat{a}_{j \alpha}^{\dagger}\left(\hat{a}_{j \alpha}\right)$ creates (annihilates) an $\alpha$ boson at site $j$. The chemical potential $\mu_{\alpha}$ already includes the out-of-plane $h_{z}$ component of the Zeeman field such that $\mu_{\uparrow}=\mu+h_{z}$ and $\mu_{\downarrow}=\mu-h_{z}$. In the second line, $\langle j, k\rangle$ sums over the nearest-neighbor sites, where the operator $\hat{\Psi}_{j}=\left(\hat{a}_{j \uparrow} \hat{a}_{j \downarrow}\right)^{T}$ denotes the boson operators collectively, H.c. is the Hermitian conjugate, $h_{y}$ is the in-plane component of the Zeeman field, and $\sigma_{y}$ is the Pauli spin matrix. In Eq. (1), we set the in-plane $h_{x}$ component of the Zeeman field to 0 without losing generality.

In Eq. (1), the spin matrices $T$ include both the spinpreserving and spin-flipping nearest-neighbor tunnelings, and they can be compactly written as $T^{j, j \pm \hat{x}}=t \sigma_{0} \pm i \gamma_{x} \sigma_{y}$ for hoppings in the $\pm x$ direction and $T^{j, j \pm \hat{y}}=t \sigma_{0} \mp i \gamma_{y} \sigma_{x}$ for hoppings in the $\pm y$ direction, where $t$ is the strength of the usual single-particle tunneling with $\sigma_{0}$ the identity matrix, and the parameters $\gamma_{x} \geqslant 0$ and $\gamma_{y} \geqslant 0$ characterize the strength and symmetry of the SOC. These spin matrices can be derived from a non-Abelian gauge field $\vec{A}=\left(\beta_{x} \sigma_{y},-\beta_{y} \sigma_{x}, 0\right)$, where $\beta_{x}$ and $\beta_{y}$ are constants in space, using the Peierls substitution. This leads to $T^{j k}=t_{0} e^{i \int_{j}^{k} \vec{A} \cdot d \vec{r}}$, such that $T^{j, j \pm \hat{x}}=$ $t_{0} \cos \beta_{x} \sigma_{0} \pm i t_{0} \sin \beta_{x} \sigma_{y}$ for tunnelings in the $\pm x$ direction and $T^{j, j \pm \hat{y}}=t_{0} \cos \beta_{y} \sigma_{0} \mp i t_{0} \sin \beta_{y} \sigma_{x}$ for tunnelings in the $\pm y$ direction. Thus, our model parameters in Eq. (1) are related to the parameters of the gauge field $\vec{A}$ via $\gamma_{x}=t \tan \beta_{x}$ and $\gamma_{y}=t \tan \beta_{y}$. Note that the ratio of $\gamma_{x}$ and $\gamma_{y}$ determines the symmetry of the SOC, and we compare and discuss three distinct limits throughout this paper: (i) Rashba SOC where $\gamma_{x}=\gamma_{y}=\gamma_{R} \neq 0$, (ii) $\operatorname{ERD}_{x}$ SOC where $\gamma_{x} \neq 0$ and $\gamma_{y}=0$, and (iii) $\mathrm{ERD}_{y}$ SOC where $\gamma_{x}=0$ and $\gamma_{y} \neq 0$.

It is very difficult to obtain the exact solutions for the model Hamiltonian given in Eq. (1) even in the absence of intercomponent interaction, SOC, and Zeeman field. Therefore, hoping to produce qualitatively accurate ground-state phases and phase diagrams, next we propose a properly generalized variational Gutzwiller ansatz for our model.

\section{B. Variational Gutzwiller ansatz}

The variational Gutzwiller ansatz for the approximate many-body wave function $|\psi\rangle$ is a product state that is formed by multiplying local ground states $\left|\psi^{j}\right\rangle$ of the entire lattice, i.e., $|\psi\rangle=\prod_{j}\left|\psi^{j}\right\rangle$, and thus it neglects the off-site correlations by construction. The simpler versions of this ansatz have been frequently used in the literature to approximate the groundstate wave functions of Bose-Hubbard-type Hamiltonians at zero temperature. In the single-component case, since the ansatz reproduces (by construction) the exact ground states of the system in the extremely strong (i.e., deep in the MI phase) and extremely weak (i.e., deep in the SF phase) interaction limits, it naturally works qualitatively well in between for the MI-SF phase-transition boundary. Earlier works also showed that the results obtained from this ansatz precisely match those of the mean-field decoupling approximation for the MI-SF phase-transition boundary, and, therefore, the level of approximation (i.e., negligence of the off-site correlations) is considered to be exactly equivalent in both methods $[4,19,20]$.

The generalized Gutzwiller wave function for the model Hamiltonian given in Eq. (1) can be written as

$$
|\psi\rangle=\prod_{j}\left(\sum_{l_{\uparrow} l_{\downarrow}} f_{l_{\uparrow} l_{\downarrow}}^{j}\left|l_{\uparrow}, l_{\downarrow}\right\rangle_{j}\right),
$$


where the complex variational parameter $f_{l_{\uparrow} l_{\downarrow}}^{j}$ determines the probability amplitude of the occupation of the Fock state $\left|l_{\uparrow}, l_{\downarrow}\right\rangle_{j}$ at site $j$. Here, the local Fock state is characterized by the occupation of $\left(l_{\uparrow}, l_{\downarrow}\right)$ bosons from each type, where $l_{\alpha}=0,1, \ldots, l_{\max }$ and $l_{\max }$ is the maximum number of $\alpha$ bosons allowed in the numerics (to be specified in Sec. III). The normalization of the wave function $\langle\psi \mid \psi\rangle=1$ requires $\sum_{l_{\uparrow} l_{\downarrow}}\left|f_{l_{\uparrow} l_{\downarrow}}^{j}\right|^{2}=1$ for each site $j$.

Given the ground-state ansatz, it is a straightforward task to calculate any of the desired observables. For instance, we are interested in the average number of local $\alpha$ bosons $N_{j \alpha}=$ $\left\langle\psi\left|\hat{n}_{j \alpha}\right| \psi\right\rangle$ and the projections of average local polarizations $P_{j q}=\left\langle\psi\left|\hat{\Psi}_{j}^{\dagger} \sigma_{q} \hat{\Psi}_{j}\right| \psi\right\rangle$ along the $q \equiv(x, y, z)$ direction. Using Eq. (2), and after some algebra, we obtain

$$
\begin{gathered}
N_{j}=\sum_{l_{\uparrow} l_{\downarrow}}\left(\left|f_{l_{\uparrow} l_{\downarrow}}^{j}\right|^{2} l_{\uparrow}+\left|f_{l_{\uparrow} l_{\downarrow}}^{j}\right|^{2} l_{\downarrow}\right), \\
P_{j z}=\sum_{l_{\uparrow} l_{\downarrow}}\left(\left|f_{l_{\uparrow} l_{\downarrow}}^{j}\right|^{2} l_{\uparrow}-\left|f_{l_{\uparrow} l_{\downarrow}}^{j}\right|^{2} l_{\downarrow}\right), \\
P_{j y}=2 \operatorname{Im} \sum_{l_{\uparrow} l_{\downarrow}} f_{l_{\uparrow} l_{\downarrow}}^{j *} f_{l_{\uparrow}-1, l_{\downarrow}+1}^{j} \sqrt{l_{\uparrow}\left(l_{\downarrow}+1\right)},
\end{gathered}
$$

where $N_{j}=N_{j \uparrow}+N_{j \downarrow}$ is the total average number of bosons on site $j$ and $P_{j z}=N_{j \uparrow}-N_{j \downarrow}$. Here, $\operatorname{Im}[\cdot]$ is the imaginary part of [·], and the real part of the same sum gives $P_{j x}$. Note that while the overall $x$ component of the average polarization $\sum_{j} P_{j x}=0$, since we already set $h_{x}=0$ in Eq. (1), SOC may still induce local $P_{j x} \neq 0$, causing Skyrmion-like spin textures [48]. As discussed in Sec. III, all of our numerical calculations show that average particle numbers are uniform across the entire lattice, and, hence, we also define $N=N_{j}$ and $N_{\alpha}=N_{j \alpha}$ for all $j$.

In order to distinguish the SF and non-SF (e.g., MI) ground states of the system, the local average number and polarization given by Eqs. (3)-(5) need to be solved self-consistently with the local single-particle and/or single-hole SF order parameters $\Delta_{j \alpha}=\left\langle\psi\left|\hat{a}_{j \alpha}\right| \psi\right\rangle$. Note that exotic SF phases involving multiparticle and/or multihole excitations are not accessible with this definition, and they are not our main interest in this work (see also Sec. IV). Using Eq. (2), and after some algebra, we obtain

$$
\begin{aligned}
\Delta_{j \uparrow} & =\sum_{l_{\uparrow} l_{\downarrow}} f_{l_{\uparrow} l_{\downarrow}}^{j *} f_{l_{\uparrow}+1, l_{\downarrow}}^{j} \sqrt{l_{\uparrow}+1}, \\
\Delta_{j \downarrow} & =\sum_{l_{\uparrow} l_{\downarrow}} f_{l_{\uparrow} l_{\downarrow}}^{j *} f_{l_{\uparrow}, l_{\downarrow}+1}^{j} \sqrt{l_{\downarrow}+1},
\end{aligned}
$$

which are complex numbers in general. As discussed in Sec. III, all of our numerical results showed that while the magnitudes of these parameters are uniform across the entire lattice, their phases are nonuniform in general, i.e., $\theta_{j \alpha}=\arg \left(\Delta_{j \alpha}\right)$ are not equal for all $j$. In this paper, we set the phase of the $\uparrow$ order parameter on some reference lattice site (which is labeled throughout this paper as $j \equiv 0$ ) to 0 , i.e., $\theta_{0 \uparrow}=0$, and define all of the remaining $\theta_{j \alpha}$ with respect to this reference site. Thus, in Sec. III, we define $\Delta_{j \alpha}=\bar{\Delta}_{\alpha} e^{i \theta_{j \alpha}}$, and distinguish the SF phases from the MI ones by looking at whether the minimum-energy configuration has $\bar{\Delta}_{\alpha} \neq 0$ or 0 . In addition, we distinguish the uniform-SF phase from nonuniform-SF ones based on whether or not the minimum-energy configuration has a uniform $\theta_{j \alpha}=\theta_{\alpha}$ for all $j$. Note that, depending on the model parameters, we may have $\bar{\Delta}_{\alpha}=0$ and $\bar{\Delta}_{-\alpha} \neq 0$, so that the ground state is a mixture of $\alpha$-MI and $(-\alpha)$-SF, where $(-\uparrow) \equiv \downarrow$, and vice versa.

In the self-consistency given by Eqs. (3)-(7), the set of variational parameters $\left\{f_{l_{\uparrow} l_{\downarrow}}\right\}$ is determined by minimizing the ground-state energy of the system. For this purpose, we solve the Schrödinger equation, i.e., $\langle\psi|H| \psi\rangle=i \hbar\langle\psi|\partial| \psi\rangle / \partial \tau$, where we set $f_{l_{\uparrow} l_{\downarrow}}^{j}(\tau)=f_{l_{\uparrow} l_{\downarrow}}^{j} e^{-i E_{0} \tau / \hbar}$, with $E_{0}$ the local groundstate energy of the system and $\tau$ the time. Using Eq. (2), and after some algebra, we obtain

$$
\begin{aligned}
& E_{0} f_{l_{\uparrow} l_{\downarrow}}^{j} \\
& =f_{l_{\uparrow} l_{\downarrow}}^{j}\left\{U_{\uparrow \downarrow} l_{\uparrow} l_{\downarrow}+\sum_{\alpha}\left[\frac{U_{\alpha \alpha}}{2} l_{\alpha}\left(l_{\alpha}-1\right)-\mu_{\alpha} l_{\alpha}\right]\right\} \\
& \quad-\sum_{\alpha, k_{j}}\left[\Delta_{k \alpha}\left(T_{\uparrow \alpha}^{j k} \sqrt{l_{\uparrow}} f_{l_{\uparrow}-1, l_{\downarrow}}^{j}+T_{\downarrow \alpha}^{j k} \sqrt{l_{\downarrow}} f_{l_{\uparrow}, l_{\downarrow}-1}^{j}\right)\right. \\
& \left.\quad+\Delta_{k \alpha}^{*}\left(T_{\uparrow \alpha}^{j k *} \sqrt{l_{\uparrow}+1} f_{l_{\uparrow}+1, l_{\downarrow}}^{j}+T_{\downarrow \alpha}^{j k *} \sqrt{l_{\downarrow}+1} f_{l_{\uparrow}, l_{\downarrow}+1}^{j}\right)\right] \\
& \quad+i h_{y}\left[\sqrt{l_{\uparrow}\left(l_{\downarrow}+1\right)} f_{l_{\uparrow}-1, l_{\downarrow}+1}^{j}-\sqrt{\left(l_{\uparrow}+1\right) l_{\downarrow}} f_{l_{\uparrow}+1, l_{\downarrow}-1}^{j}\right],
\end{aligned}
$$

where $k_{j}$ sums over the nearest neighbors $k$ of site $j$. We note that all of the tunneling and SOC terms vanish in the MI phase when $\bar{\Delta}_{\alpha}=0$, and, therefore, recently proposed magnetic (spin-textured) MI phases [36-40] are not accessible within our Gutzwiller ansatz. However, the method may still give a quantitatively accurate description of the MI-SF phasetransition boundary as well as the nonuniform-SF phases near this boundary. To understand the competition between the interaction, tunneling, SOC, and Zeeman field, and the resultant MI and SF phases, let us first discuss the classical limit and analyze the ground-state phase diagram of the system in the atomic limit.

\section{Atomic limit: MI phases}

Setting $t=\gamma_{x}=\gamma_{y}=0$ in the Hamiltonian decouples all of the lattice sites from each other, and, therefore, it is sufficient to consider a single site to understand the resultant MI phases. First of all, in contrast with the $h_{y}=0$ case where $N_{\alpha}$ is conserved for both $\alpha$ bosons, only the total number $N=N_{\uparrow}+N_{\downarrow}$ of bosons is a good quantum number when $h_{y} \neq 0$. Thus, the MI lobes must be labeled accordingly. Using Gutzwiller-like local ground states $\left|\psi_{N}\right\rangle=\sum_{l_{\uparrow} l_{\downarrow} \ni l_{\uparrow}+l_{\downarrow}=N} f_{l_{\uparrow} l_{\downarrow}}\left|l_{\uparrow}, l_{\downarrow}\right\rangle$, which can be shown to be exact for a given total particle sector $N$, we can easily obtain the exact local ground-state energy $\mathcal{E}_{N}=\left\langle\psi_{N}|H| \psi_{N}\right\rangle$ of the system by minimizing $\mathcal{E}_{N}$ with respect to $f_{l_{\uparrow} l_{\downarrow}}$.

For instance, $\mathcal{E}_{0}=0$ in the trivial case when $N=0$, and its corresponding eigenstate is the vacuum state $|0,0\rangle$ with $f_{00}=1$. There are two energy eigenvalues when $N=1$, and 
$\mathcal{E}_{1}$ can be written as

$$
\mathcal{E}_{1}=\Phi_{1}^{\dagger}\left(\begin{array}{cc}
-\mu_{\uparrow} & i h_{y} \\
-i h_{y} & -\mu_{\downarrow}
\end{array}\right) \Phi_{1},
$$

where $\Phi_{1}=\left(f_{10} f_{01}\right)^{T}$. Likewise, there are three energy eigenvalues when $N=2$, and $\mathcal{E}_{2}$ can be written as

$$
\mathcal{E}_{2}=\Phi_{2}^{\dagger}\left(\begin{array}{ccc}
-2 \mu+U_{\uparrow \downarrow} & -i \sqrt{2} h_{y} & i \sqrt{2} h_{y} \\
i \sqrt{2} h_{y} & -2 \mu_{\uparrow}+U_{\uparrow \uparrow} & 0 \\
-i \sqrt{2} h_{y} & 0 & -2 \mu_{\downarrow}+U_{\downarrow \downarrow}
\end{array}\right) \Phi_{2},
$$

where $\Phi_{2}=\left(f_{11} f_{20} f_{02}\right)^{T}$. All of the energy eigenvalues and their corresponding eigenstates can be easily obtained by diagonalizing such matrices for any given $N$, and $E_{0}$ corresponds to the minimal eigenvalue.

(a) $h_{y}=0$

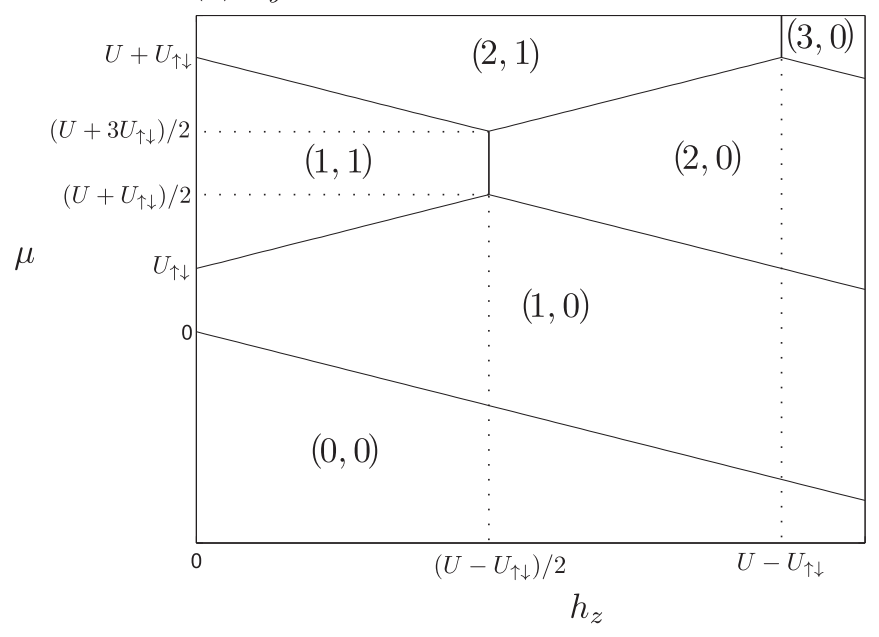

(b) $h_{z}=0 \longrightarrow, h_{z}=0.1 \mathrm{U}---$

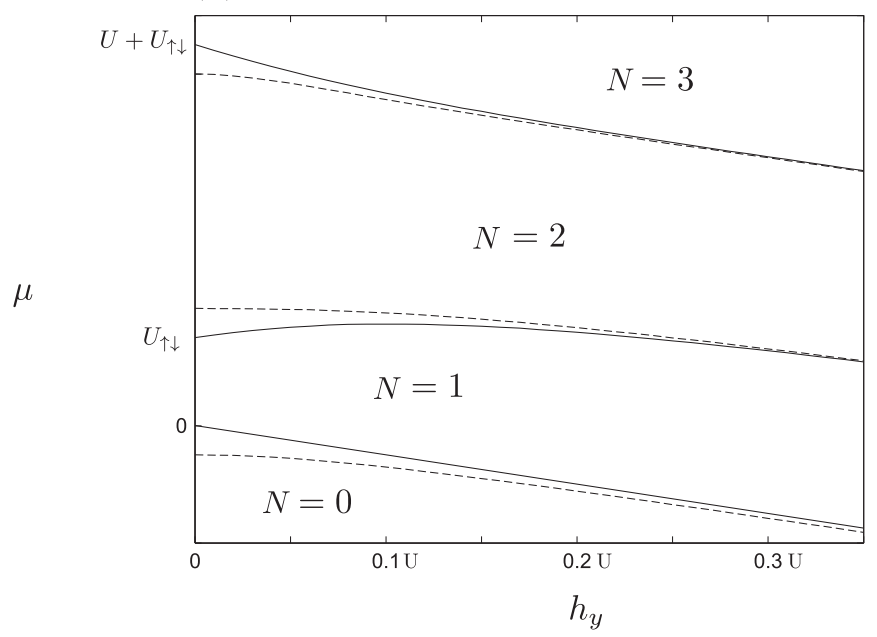

FIG. 1. The atomic-limit $\left(t=\gamma_{x}=\gamma_{y}=0\right)$ phase diagrams are shown as functions of (a) $\mu$ and $h_{z}$ for $h_{y}=0$, and (b) $\mu$ and $h_{y}$ for $h_{z}=0$ (solid line) and $h_{z}=0.1 U$ (dashed line). The MI lobes are labeled by (a) $\left(N_{\uparrow}, N_{\downarrow}\right)$ and (b) $N=N_{\uparrow}+N_{\downarrow}$. While we set $U_{\uparrow \downarrow}=0.3 U$ in these figures, they are schematically correct as long as $0<U_{\uparrow \downarrow}<U=U_{\uparrow \uparrow}=U_{\downarrow \downarrow}$.
In Fig. 1, we present the atomic-limit phase diagrams as functions of $\mu$ and $h_{z}$ for $h_{y}=0$ [Fig. 1(a)], and $\mu$ and $h_{y}$ for $h_{z}=0$ and $h_{z}=0.1 U$ [Fig. 1(b)]. The MI lobes are naturally labeled by $\left(N_{\uparrow}, N_{\downarrow}\right)$ in Fig. 1(a) and $N$ in Fig. 1(b) as explained above. While we set $U_{\uparrow \downarrow}=0.3 U$ in these figures, they are schematically correct as long as $0<U_{\uparrow \downarrow}<U=U_{\uparrow \uparrow}=U_{\downarrow \downarrow}$. When $h_{y}=0$, Fig. 1(a) shows that the size of the $N=1$ lobe grows as $h_{z}$ increases toward $\left(U-U_{\uparrow \downarrow}\right) / 2$ and its size remains essentially unchanged for $h_{z}>\left(U-U_{\uparrow \downarrow}\right) / 2$. This is in contrast with the $N=2$ MI lobe, the size of which shrinks as $h_{z}$ increases toward $\left(U-U_{\uparrow \downarrow}\right) / 2$, followed by an increase between $\left(U-U_{\uparrow \downarrow}\right) / 2<h_{z}<U-U_{\uparrow \downarrow}$, and then its size remains essentially unchanged for $h_{z}>U-U_{\uparrow \downarrow}$. Similarly, when $h_{z}=0$, Fig. 1(b) shows that $h_{y}$ has a similar effect on the sizes of the MI lobes. Having established the theoretical formalism, next we present the details of our numerical calculations.

\section{NUMERICAL RESULTS}

First of all, we need to solve Eq. (8) self-consistently with Eqs. (6) and (7) for the eigenstates of the lowest-energy eigenvalue. This can be achieved via the iterative method of relaxation as follows: (i) first, start with an input set of $\left\{\Delta_{j \alpha}\right\}$, (ii) next, construct the Hamiltonian matrix given in Eq. (8), (iii) then, use the lowest-energy eigenstates in Eqs. (6) and (7) and generate a new set of $\left\{\Delta_{j \alpha}\right\}$, and (iv) finally, repeat these steps until the input and output sets of $\left\{\Delta_{j \alpha}\right\}$ lie within a confidence level. Once the iterative method converges, we use Eqs. (3)-(5) to calculate the local average number of bosons and their polarizations.

As we emphasized in Sec. IIB, while the Gutzwiller ansatz does not tell anything about the possibility of having magnetic spin textures inside the MI lobes, it may still give a quantitatively accurate description of the competition between the interaction, tunneling, SOC, and Zeeman field. In this section, we solve Eqs. (3)-(8) self-consistently near the MI-SF phase-transition boundary of the first two $(N=1$ and 2$)$ insulating lobes, and analyze how their sizes change with the strength and symmetry of the SOC and Zeeman field. For this purpose, we set the cutoff of $l_{\alpha}$ in the sums to $l_{\max }=4$, which is sufficient near the $N=1$ and $N=2 \mathrm{MI}$ lobes, and the intraparticle and interparticle interactions to $U_{\uparrow \uparrow}=U_{\downarrow \downarrow}=U$ and $U_{\uparrow \downarrow}=0.3 U$, respectively. In addition, we choose equal magnitudes for the Rashba and ERD SOCs such that $\gamma_{E}=\sqrt{2} \gamma_{R}$. It turns out that Eqs. (3)-(8) allow for many multiple solutions and, therefore, we use of the order of $10^{4}$ random initial sets of $\left\{\Delta_{j \alpha}\right\}$, and then eventually keep the one which has the lowest ground-state energy.

\section{A. SOC-induced nonuniform-SF phase}

In order to characterize the possible SF phases, we first solve the self-consistency equations on finite $M \times L$ lattices with periodic boundary conditions, but without any assumption on the symmetry of $\Delta_{j \alpha}$. By letting $\{M, L\}=\{3,4,5, \ldots, 20\}$ and using numerous combinations of SOC and Zeeman fields, we find that while the magnitudes of $\Delta_{j \alpha}$ are uniform across the entire lattice, their phases may vary from site to site due to 
SOC, such that

$$
\Delta_{j \alpha}=\bar{\Delta}_{\alpha} e^{i \theta_{j \alpha}},
$$

where $\bar{\Delta}_{\alpha}=\left|\Delta_{j \alpha}\right|$ for all $j$. This result is in agreement with an earlier study [42], and it shows that the SF phase can be nonuniform depending on the model parameters. Moreover, assuming Eq. (11) holds, we solve the self-consistency equations on very large lattices, and find that the phase $\theta_{j \alpha}$ jumps uniformly from one site to the next in $x$ and/or $y$ directions, and also that the amount of jump is the same for both $\uparrow$ and $\downarrow$ components. In other words, the equal-phase-jump configuration between nearest-neighbor sites is energetically more favorable than the repeating patterns of multiple phase jumps. Thus, our numerical calculations suggest that the phases $\theta_{j \alpha}$, in their minimum-energy configuration, obey the following pattern:

$$
\theta_{j \alpha}=\theta_{0 \alpha}+j_{x} \theta_{x}+j_{y} \theta_{y},
$$

where $\left(j_{x}, j_{y}\right)$ are $(x, y)$ coordinates of the site $j$ with respect to our reference site 0 . In this paper, we set $\theta_{0 \uparrow}=0$ without losing generality, and determine the rest of the phases, i.e., $\theta_{0 \downarrow}, \theta_{x}$, and $\theta_{y}$, with respect to it. It also turns out that $\theta_{y}=0$ for $\mathrm{ERD}_{x}$ and $\theta_{x}=0$ for $\mathrm{ERD}_{y} \mathrm{SOC}$, and $\left|\theta_{x}\right|$ and $\left|\theta_{y}\right|$ are not necessarily equal for Rashba SOC when $h_{y} \neq 0$.

Before we move on to the numerical analysis of the nonuniform-SF phases, we emphasize that $\theta_{0 \downarrow}$ may not be a gauge-independent quantity due to the mean-field definition of the SF order parameters. For illustration purposes, let us consider a lattice model with Rashba or ERD SOC (the latter can be either parallel or perpendicular to the in-plane Zeeman field) and write down its SF order parameters using the two coordinate systems shown in Fig. 2. The Hamiltonian of the system in Fig. 2(b) can be transformed to that of the system in Fig. 2(a) via the following canonical transformation: $\hat{b}_{j \uparrow}=\hat{a}_{j \uparrow}$ and $\hat{b}_{j \downarrow}=i \hat{a}_{j \downarrow}$ for all $j$. Letting $\left|\psi^{(\mathrm{a})}\right\rangle$ and $\left|\psi^{(\mathrm{b})}\right\rangle$ be the ground states of Figs. 2(a) and 2(b), respectively, and expanding $\left|\psi^{(\mathrm{a})}\right\rangle$ in the occupation number basis $\left|l_{\uparrow}, l_{\downarrow}\right\rangle^{a}$ of $a$ bosons and $\left|\psi^{(\mathrm{b})}\right\rangle$ in $\left|l_{\uparrow}, l_{\downarrow}\right\rangle^{b}$ of $b$ bosons show that the expansion coefficients are equal for the corresponding terms. Therefore, using Eq. (7), the order parameter $\Delta_{0 \downarrow}^{(a)}$ of the reference site in Fig. 2(a), $\Delta_{0 \downarrow}^{(\mathrm{a})}=\left\langle\psi^{(\mathrm{a})}\left|a_{0 \downarrow}\right| \psi^{(\mathrm{a})}\right\rangle=\left\langle\psi^{(\mathrm{b})}\left|b_{0 \downarrow}\right| \psi^{(\mathrm{b})}\right\rangle$, is related to the order parameter of the same site in Fig. 2(b) by $\Delta_{0 \downarrow}^{(\mathrm{b})}=\left\langle\psi^{(\mathrm{b})}\left|a_{0 \downarrow}\right| \psi^{(\mathrm{b})}\right\rangle=-i\left\langle\psi^{(\mathrm{b})}\left|b_{0 \downarrow}\right| \psi^{(\mathrm{b})}\right\rangle=$

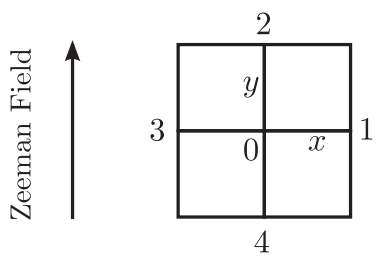

(a)

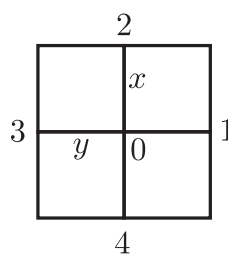

(b)
FIG. 2. The phase $\theta_{0 \downarrow}$ of the order parameter $\Delta_{0 \downarrow}$ of the reference site 0 may depend on the choice of coordinate system and is not a gauge-independent quantity within the mean-field theory. This can be seen by comparing the order parameters using the coordinate systems shown in (a) and (b) as discussed in the text. $-i \Delta_{0 \downarrow}^{(\mathrm{a})}$. This shows that $\theta_{0 \downarrow}$ depends on the coordinate system and may not be a gauge-independent quantity. However, relative phases of all of the neighboring sites, i.e., $\theta_{j \alpha}-\theta_{0 \alpha}$ for all $j$, are not affected by the above transformation, and, hence, they are gauge independent.

Equations (11) and (12) suggest that our numerical results (to be discussed below) for the phases $\theta_{0 \downarrow}, \theta_{x}$, and $\theta_{y}$ can be analytically understood by simply looking at the local ground-state energy $E_{0}=\left\langle\psi^{j}|H| \psi^{j}\right\rangle$ of the system at any particular site $j$. Note that the local Gutzwiller ground state of site $j,\left|\psi^{j}\right\rangle=\sum_{l_{\uparrow} l_{\downarrow}} f_{l_{\uparrow} l_{\downarrow}}^{j}\left|l_{\uparrow}, l_{\downarrow}\right\rangle_{j}$, can be determined by solving Eq. (8) for the minimum-energy configuration. Similar to the SF order parameters shown in Eq. (11), our numerical calculations also suggest that the magnitudes of $f_{l_{\uparrow} l_{\downarrow}}^{j}$ are uniform across the entire lattice, such that

$$
f_{l_{\uparrow} l_{\downarrow}}^{j}=\bar{f}_{l_{\uparrow} l_{\downarrow}} e^{i \phi_{l_{\uparrow} \downarrow}^{j}}
$$

where $\bar{f}_{l_{\uparrow} l_{\downarrow}}=\left|f_{l_{\uparrow} l_{\downarrow}}^{j}\right|$ for all $j$. In addition, we find that while the interaction terms compete with the the rest of the (tunneling, SOC, and in-plane Zeeman) terms in the Hamiltonian for the magnitudes $\bar{f}_{l_{\uparrow} l_{\downarrow}}$, the phases $\phi_{l_{\uparrow} l_{\downarrow}}^{j}$ are solely determined by the interplay between tunneling, SOC, and in-plane Zeeman field, in such a way as to minimize the energy $E_{0}$ for a given set of magnitudes $\bar{f}_{l_{\uparrow} l_{\downarrow}}$. Using Eqs. (11)-(13), and after some algebra, $E_{0}=\left\langle\psi^{0}|H| \psi^{0}\right\rangle$ of the reference site 0 can be written as

$$
\begin{aligned}
E_{0}= & -4 t\left(\bar{\Delta}_{\uparrow}^{2}+\bar{\Delta}_{\downarrow}^{2}\right)\left(\cos \theta_{x}+\cos \theta_{y}\right) \\
& -8 \bar{\Delta}_{\uparrow} \bar{\Delta}_{\downarrow}\left(\gamma_{y} \cos \theta_{0 \downarrow} \sin \theta_{y}-\gamma_{x} \sin \theta_{0 \downarrow} \sin \theta_{x}\right) \\
& +\sum_{l_{\uparrow} l_{\downarrow}} \bar{f}_{l_{\uparrow} l_{\downarrow}}^{2}\left\{U_{\uparrow \downarrow} l_{\uparrow} l_{\downarrow}+\sum_{\alpha}\left[\frac{U_{\alpha \alpha}}{2} l_{\alpha}\left(l_{\alpha}-1\right)-\mu_{\alpha} l_{\alpha}\right]\right\} \\
& -2 h_{y} \operatorname{Im} \sum_{l_{\uparrow} l_{\downarrow}} f_{l_{\uparrow} l_{\downarrow}}^{0 *} f_{l_{\uparrow}-1, l_{\downarrow}+1}^{0} \sqrt{l_{\uparrow}\left(l_{\downarrow}+1\right)} .
\end{aligned}
$$

Using Eq. (5), the last term can also be written as $-h_{y} P_{0 y}$. Much of our analytical understanding of the numerical calculations is based on the analysis of this expression in various limits, and we refer to it quite frequently in the remaining parts of the paper.

For example, in the simpler case of non-SF phases when $\bar{\Delta}_{\alpha}=0$, since the tunneling and SOC terms disappear from Eq. (14), the set of phases $\left\{\phi_{l_{\uparrow} l_{\downarrow}}^{j}\right\}$ is determined only by $h_{y}$. For a given set of $\left\{\bar{f}_{l_{\uparrow} l_{\downarrow}}\right\}$, the contribution of the in-plane Zeeman field to $E_{0}$ is minimum when the relative angles satisfy the condition $\phi_{l_{\uparrow}-1, l_{\downarrow}+1}^{0}-\phi_{l_{\uparrow} l_{\downarrow}}^{0}=\pi / 2$ for all $l_{\uparrow}$ and $l_{\downarrow}$ as long as $h_{y} \neq 0$.

This condition still holds in the SF phase as long as $h_{y} \neq 0$ and there is no SOC. To prove this, let us set $\gamma_{x}=\gamma_{y}=0$ in Eq. (14), in which case the contribution of the tunneling term $-4 t\left(\bar{\Delta}_{\uparrow}^{2}+\bar{\Delta}_{\downarrow}^{2}\right)\left(\cos \theta_{x}+\cos \theta_{y}\right)$ to $E_{0}$ is minimized when $\bar{\Delta}_{\uparrow}$ and $\bar{\Delta}_{\downarrow}$ are maximum and $\theta_{x}=$ $\theta_{y}=0$, recovering the usual case where the SF phase is uniform across the entire lattice. Using Eq. (6), we 

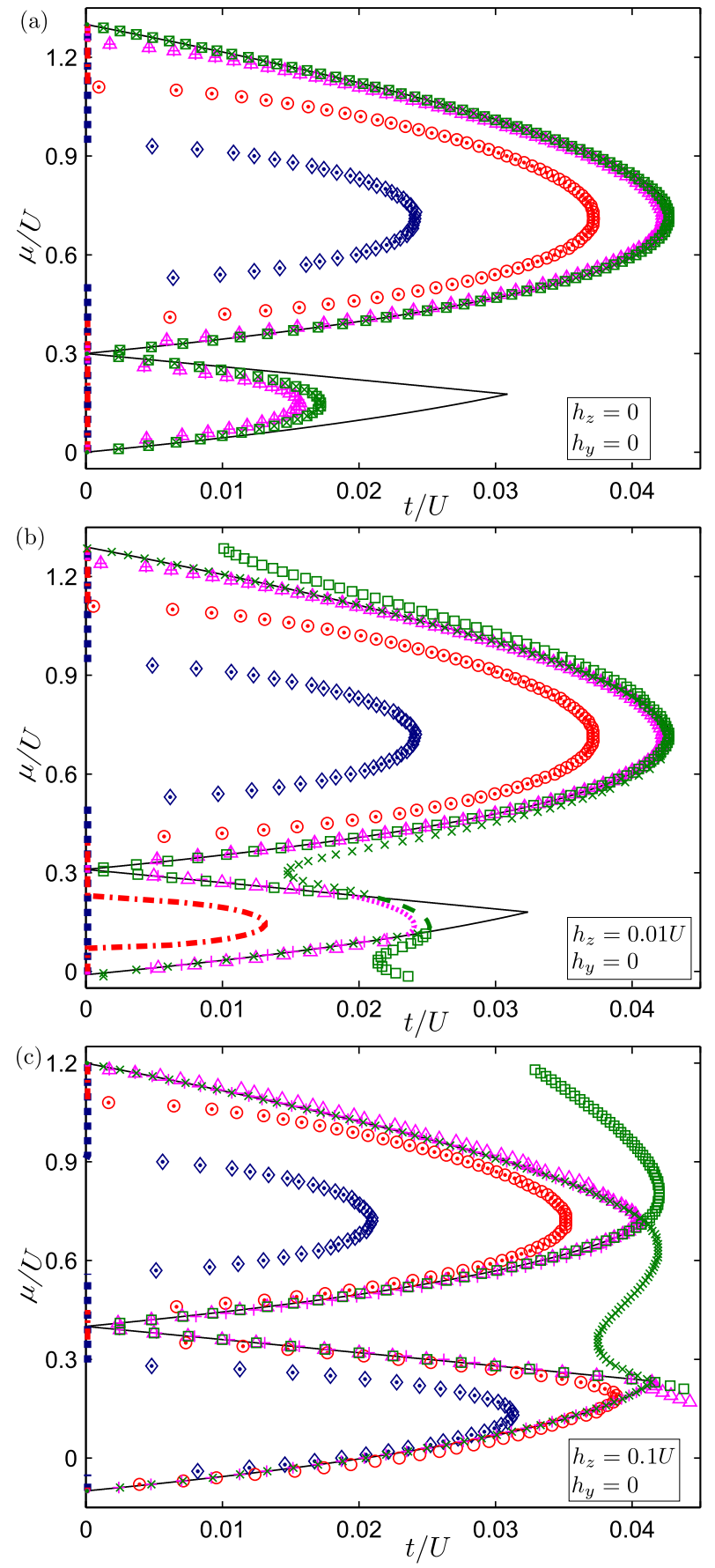

\begin{tabular}{|c|c|c|c|}
\hline 1 st order & 2nd order $\downarrow(\uparrow)$ Boson & $\operatorname{Rashba}\left(\gamma_{x}=\gamma_{y}=\gamma_{R}\right)$ & $E R D\left(\right.$ either $\gamma_{x}=\gamma_{E}$ or $\left.\gamma_{y}=\gamma_{E}\right)$ \\
\hline$\because$ & $\diamond(\cdot)$ & $\gamma_{R}=0.05 U$ & $\gamma_{E}=0.05 \sqrt{2} U$ \\
\hline שי = & $\circ(\cdot)$ & $\gamma_{R}=0.03 U$ & $\gamma_{E}=0.03 \sqrt{2} U$ \\
\hline .....! & $\Delta(+)$ & $\gamma_{R}=0.01 U$ & $\gamma_{E}=0.01 \sqrt{2} U$ \\
\hline-- & (x) & $\gamma_{R}=0$ & $\gamma_{E}=0$ \\
\hline
\end{tabular}

FIG. 3. (Color online) Ground-state phase diagrams with out-of-plane $h_{z}$ Zeeman fields. The MI-SF phase-transition boundaries are shown as functions of $\mu$ and $t$ for the first two MI lobes, i.e., $N=1$ and 2, where we consider (a)-(c) Rashba SOC and (d)-(f) ERD SOC. Here, we set $h_{y}=0, U_{\uparrow \uparrow}=U_{\downarrow \downarrow}=U$, and $U_{\uparrow \downarrow}=0.3 U$ in all figures. In addition, the black solid lines are guides to the eye, which are obtained from Eq. (15) (see the text for details).

have $\Delta_{0 \uparrow}=\sum_{l_{\uparrow} l_{\downarrow}} \bar{f}_{l_{\uparrow} l_{\downarrow}} \bar{f}_{l_{\uparrow}+1, l_{\downarrow}} e^{i\left(\phi_{l_{\uparrow}+1, l_{\downarrow}}^{0}-\phi_{l_{\uparrow} l_{\downarrow}}^{0}\right)} \sqrt{l_{\uparrow}+1}$, which is chosen to be a real number in this paper, but $\Delta_{0 \downarrow}=$ $\sum_{l_{\uparrow} l_{\downarrow}} \bar{f}_{l_{\uparrow} l_{\downarrow}} \bar{f}_{l_{\uparrow}, l_{\downarrow}+1} e^{i\left(\phi_{l_{\uparrow}, l_{\downarrow}+1}^{0}-\phi_{l_{\uparrow} l_{\downarrow}}^{0}\right)} \sqrt{l_{\downarrow}+1}$ is a complex number
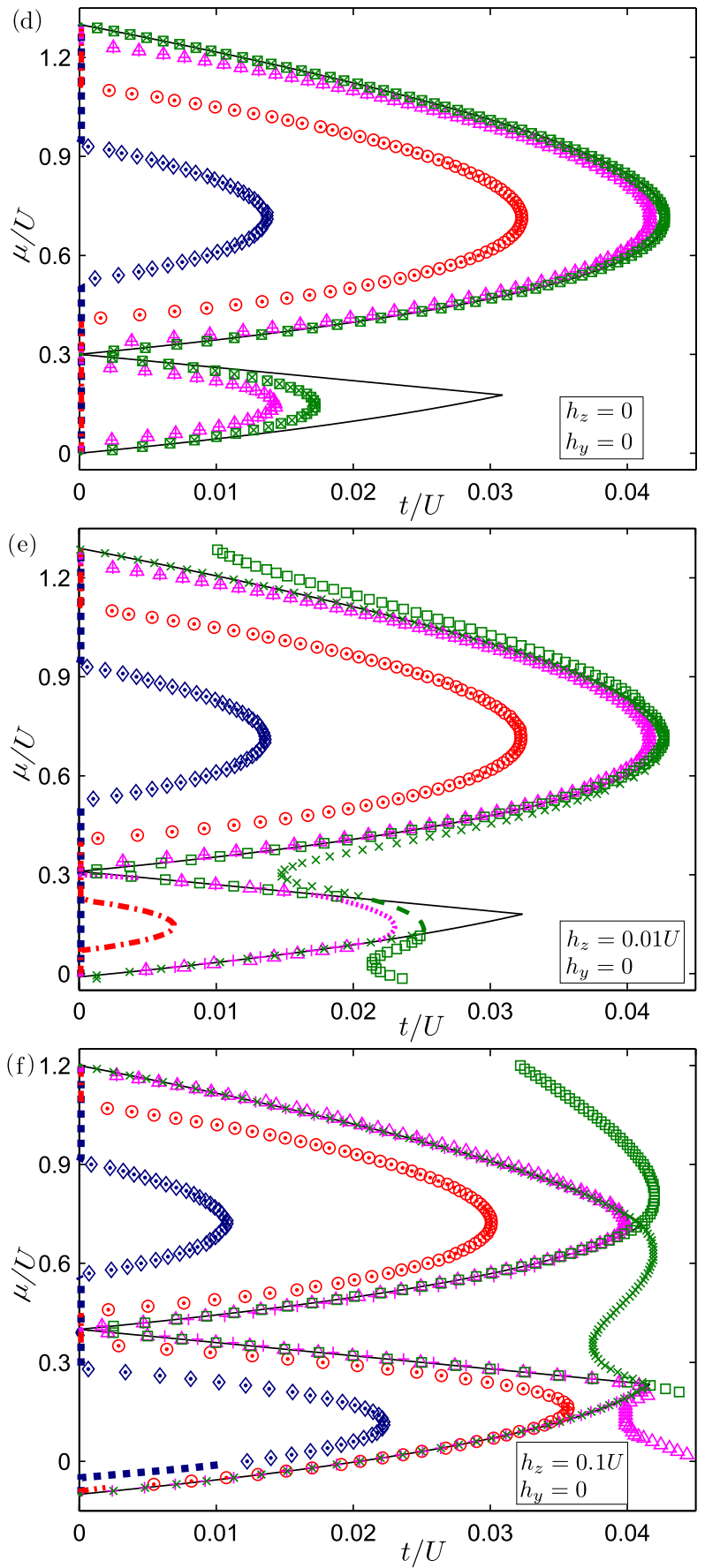

$\begin{aligned} \gamma_{R} & =0.05 U \\ \gamma_{R} & =0.03 U \\ \gamma_{R} & =0.01 U \\ \gamma_{R} & =0\end{aligned}$

$$
\begin{aligned}
\gamma_{E} & =0.05 \sqrt{2} U \\
\gamma_{E} & =0.03 \sqrt{2} U \\
\gamma_{E} & =0.01 \sqrt{2} U
\end{aligned}
$$


we already set $\theta_{0 \uparrow}=0$ in this paper, $\phi_{l_{\uparrow}+1, l_{\downarrow}}^{0}-\phi_{l_{\uparrow} l_{\downarrow}}^{0}=0$ maximizes the order parameters. It is important to note that a set of phases can simultaneously satisfy both this condition and the condition $\phi_{l_{\uparrow}-1, l_{\downarrow}+1}^{0}-\phi_{l_{\uparrow} l_{\downarrow}}^{0}=\pi / 2$ that minimizes the in-plane Zeeman term, and combining these two conditions reveals that $\phi_{l_{\uparrow}, l_{\downarrow}+1}^{0}-\phi_{l_{\uparrow} l_{\downarrow}}^{0}=\pi / 2$ for all $l_{\uparrow}$ and $l_{\downarrow}$. This, in turn, implies that $\theta_{0 \downarrow}=\pi / 2$, which is in agreement with our numerical results.

For completeness, here we find it is useful to comment on the effects of an in-plane $h_{x}$ Zeeman field. If such a field is considered in Eq. (1), its contribution to $E_{0}$ can be explicitly written as $-2 h_{x} \operatorname{Re} \sum_{l_{\uparrow} l_{\downarrow}} f_{l_{\uparrow} l_{\downarrow}}^{0 *} f_{l_{\uparrow}-1, l_{\downarrow}+1}^{0} \sqrt{l_{\uparrow}\left(l_{\downarrow}+1\right)}$, which is nothing but $-h_{x} P_{0 x}$. Assuming $h_{y}=0$, and using similar arguments as above, we find $\theta_{0 \downarrow}=0$ in this case, which is again in agreement with our numerical results. Note that this analysis is also consistent with our previous discussion about the relation between the order parameters $\Delta_{0 \downarrow}^{(\mathrm{b})}=-i \Delta_{0 \downarrow}^{(\mathrm{a})}$ that are defined using the coordinate systems shown in Fig. 2. Next, we are ready to analyze the effects of the strength and symmetry of the SOC and Zeeman field on the nonuniform-SF phase and resultant phase diagrams.

\section{B. MI-SF phase transitions: Out-of-plane Zeeman field}

We set $h_{y}=0$ in this section, and study Rashba and ERD SOCs with an out-of-plane Zeeman field. We recall that the SF phases in this work are distinguished from the MI ones by their finite $\bar{\Delta}_{\uparrow}$ and/or $\bar{\Delta}_{\downarrow}$ order parameters, and, therefore, single-particle and/or single-hole excitations are always gapped inside the MI lobes. However, since our definition of the SF order parameters does not discriminate the possibility of exotic multiparticle and/or multihole excitations that may be gapless, our single-particle and/or single-hole MI lobes may still have some sort of hidden (exotic) SF orders. The fate of such exotic SF phases is beyond the scope of this work, and they deserve a separate analysis of their own (see also Sec. IV).

In Figs. 3(a) and 3(d), we show the $h_{z}=0$ ground-state phase diagrams as functions of $\mu$ and $t$ for Rashba and ERD SOCs, respectively. Since $U_{\uparrow \uparrow}=U_{\downarrow \downarrow}$ and $\mu_{\uparrow}=\mu_{\downarrow}$ in these figures, the order parameters must also be equal, $\bar{\Delta}_{\uparrow}=\bar{\Delta}_{\downarrow}$, and, therefore, both $\alpha$ components simultaneously undergo MI-SF transition across the phase-transition boundary. In particular, the $N=2 \mathrm{MI}$ lobe is characterized by $N_{\uparrow}=N_{\downarrow}=$ 1 and all of its elementary excitations are gapped. However, the $N=1$ lobe is proposed to have an exotic counterflow-SF order of particle-hole pairs as discussed in the literature when there is no SOC [24,25,29,31]. These figures clearly show that the sizes of the MI lobes shrink as a function of increasing SOC strength in both Rashba and ERD cases, which is a result of increased mobility of the particles due to SOC tunneling. Note in Figs. 3(a) and 3(d) that the $N=1$ MI lobe shrinks so much that it lives right on the $\mu$ axis for sufficiently strong SOC, and the system becomes a SF even in the $t / U \rightarrow 0$ limit.

In Fig. 3 (and also the ones below), the black solid lines are guides to the eye and represent the MI-SF phase-transition boundary between the $\left(N_{\uparrow}, N_{\downarrow}\right)$ MI lobes and uniform-SF phase when there is no SOC and in-plane Zeeman field. Setting $\gamma_{x}=\gamma_{y}=0$ and $h_{y}=0$ in Eq. (1), the mean-field
MI-SF phase-transition boundary can easily be obtained within the decoupling approximation, leading to the analytical expression [30]

$$
\begin{aligned}
\mu_{\alpha}^{p, h}= & U_{\alpha \alpha}\left(N_{\alpha}-1 / 2\right)+U_{\uparrow \downarrow} N_{-\alpha}-2 t \\
& \pm \sqrt{U_{\alpha \alpha}^{2} / 4-U_{\alpha \alpha}\left(4 N_{\alpha}+2\right) t+4 t^{2}}
\end{aligned}
$$

where $\alpha \equiv(\uparrow, \downarrow)$ labels the transition from $\alpha$-MI to $\alpha$-SF, and $(p, h)$ together with \pm signs correspond to the particle and hole branches, respectively. Here, $(-\uparrow) \equiv \downarrow$, and vice versa. Note that when $U_{\uparrow \downarrow}=0$, Eq. (15) reduces to two independent copies of the usual mean-field result for the single-component model. We use $\mu_{\uparrow}^{h}$ for the transition from $(1,0)$ MI to $\uparrow-$ SF by removing one $\uparrow$ boson, $\mu_{\downarrow}^{p}$ for the transition from $(1,0) \mathrm{MI}$ to $\downarrow$-SF by adding one $\downarrow$ boson, $\mu_{\downarrow}^{h}$ for the transition from $(1,1)$ MI to $\downarrow$-SF by removing one $\downarrow$ boson, $\mu_{\uparrow}^{p}$ for the transition from $(1,1) \mathrm{MI}$ to $\uparrow-\mathrm{SF}$ by adding one $\uparrow$ boson, etc. However, this expression is not applicable to the $N=1 \mathrm{MI}$ lobe when there is no SOC and Zeeman field, and this is clearly seen in Figs. 3(a) and 3(d).

In Figs. 3(b) and 3(e), a relatively small $h_{z}=0.01 U$ is added to the parameters of Figs. 3(a) and 3(d), breaking the degeneracy between $\uparrow$ and $\downarrow$ bosons. Although this leads to a slight shift in $\mu_{\uparrow}$ and $\mu_{\downarrow}$, it has dramatic consequences on the ground-state phase diagrams. First of all, unlike the $h_{z}=0$ case, the $\uparrow$ and $\downarrow$ bosons do not simultaneously become SF, unless the spin-mixing SOC strength is sufficiently strong and makes up for the chemical-potential asymmetry caused by $h_{z} \neq 0$. For instance, near the particle (hole) branch of the $N=1 \mathrm{MI}$ lobe, it is the $\downarrow$ ( $\uparrow)$ component which first becomes a $\downarrow$-SF ( $\uparrow-S F$ ) as a function of increasing $t$. Second, unlike the $h_{z}=0$ case, the $N=1 \mathrm{MI}$ lobe becomes a $(1,0) \mathrm{MI}$, and, therefore, its SF transition boundary gradually converges to that given by Eq. (15) for small $t / U$ values. Third, unlike the $h_{z}=0$ case, we find regions of first-order MI-SF phase transitions near the tip of the $N=1$ lobe, and this explains why the boundary given by our numerical calculations and Eq. (15) has an increasing mismatch for large $t / U$ values.

When $h_{z}$ is increased to $h_{z}=0.1 U$, as shown in Figs. 3(c) and 3(f), the first-order transition regions shrink near to the very tip of the $N=1 \mathrm{MI}$ lobe, and, therefore, Eq. (15) provides better matches with our numerical results. We also note that while the size of the $N=1 \mathrm{MI}$ lobe grows with increasing $h_{z}$, the size of the $N=2 \mathrm{MI}$ lobe shrinks. We find that such trends are independent of SOC strength, and, therefore, they are in good qualitative agreement with what is expected from Eq. (15). Furthermore, since these trends are also strongly correlated with the sizes of the MI lobes in the atomic limit, Fig. 1 provides a rough intuition about how the sizes of the MI lobes change as a function of $h_{z}$.

In addition, we see in Fig. 3 that ERD SOC shrinks the sizes of MI lobes a little bit more than Rashba SOC, and this small difference may be understood from Eq. (14) as follows. For a given set of $\left\{\bar{f}_{l_{\uparrow} l_{\downarrow}}\right\}$, contribution of the tunneling term to $E_{0}$ is minimized when $\bar{\Delta}_{\uparrow}$ and $\bar{\Delta}_{\downarrow}$ are maximum, and this can best be achieved if $\phi_{l_{\uparrow}+1, l_{\downarrow}}^{0}-\phi_{l_{\uparrow} l_{\downarrow}}^{0}=$ const $=\theta_{0 \uparrow}$ and $\phi_{l_{\uparrow}, l_{\downarrow}+1}^{0}-\phi_{l_{\uparrow} l_{\downarrow}}^{0}=\mathrm{const}=\theta_{0 \downarrow}$ for all $l_{\uparrow}$ and $l_{\downarrow}$. Recall that the former phase is set to 0 in this paper [see the discussion below 
Eq. (12)]. Minimizing the contribution of Rashba SOC terms to $E_{0}$, we find fourfold degenerate solutions:

\begin{tabular}{lcccc}
\hline \hline & (i) & (ii) & (iii) & (iv) \\
\hline$\theta_{0 \downarrow}=$ & $\pi / 4$ & $3 \pi / 4$ & $-3 \pi / 4$ & $-\pi / 4$ \\
$\theta_{x} \in$ & {$[-\pi / 2,0)$} & {$[-\pi / 2,0)$} & $(0, \pi / 2]$ & $(0, \pi / 2]$ \\
$\theta_{y} \in$ & $(0, \pi / 2]$ & {$[-\pi / 2,0)$} & {$[-\pi / 2,0)$} & $(0, \pi / 2]$ \\
\hline \hline
\end{tabular}

where the semiopen intervals are due to nonzero SOC. On the other hand, since the ERD SOC breaks the rotational symmetry, we set $\theta_{x}=\theta_{E}$ and $\theta_{y}=0$ for $\mathrm{ERD}_{x}$, and $\theta_{y}=\theta_{E}$

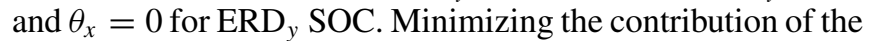
$\mathrm{ERD}_{x}$ SOC term to $E_{0}$, we find twofold degenerate solutions: (i) $\theta_{E} \in(0, \pi / 2]$ together with $\theta_{0 \downarrow}=\pi / 2$, and (ii) $\theta_{E} \in$ $[-\pi / 2,0)$ together with $\theta_{0 \downarrow}=-\pi / 2$. Similarly, minimizing the contribution of the $\mathrm{ERD}_{y} \mathrm{SOC}$ term to $E_{0}$, we again find twofold degenerate solutions: (i) $\theta_{E} \in(0, \pi / 2]$ together with $\theta_{0 \downarrow}=0$, and (ii) $\theta_{E} \in[-\pi / 2,0)$ together with $\theta_{0 \downarrow}=\pi$. Based on this analysis, the tunneling and SOC contributions to $E_{0}$ can be written as $-8 t\left(\bar{\Delta}_{\uparrow}^{2}+\bar{\Delta}_{\downarrow}^{2}\right) \cos \theta_{R}-8 \bar{\Delta}_{\uparrow} \bar{\Delta}_{\downarrow} \gamma_{R} \sqrt{2} \sin \theta_{R}$ for the Rashba SOC where $\left|\theta_{x}\right|=\left|\theta_{y}\right|=\theta_{R} \in(0, \pi / 2]$, and $-4 t\left(\bar{\Delta}_{\uparrow}^{2}+\bar{\Delta}_{\downarrow}^{2}\right)\left(1+\cos \theta_{E}\right)-8 \bar{\Delta}_{\uparrow} \bar{\Delta}_{\downarrow} \gamma_{E} \sin \left|\theta_{E}\right|$ for the ERD SOC. Setting $\gamma_{E}=\sqrt{2} \gamma_{R}$ as in our numerical calculations, these expressions show that the contribution of ERD SOC to $E_{0}$ is always smaller than that of Rashba SOC, which, in turn, implies that the ERD SOC gives way to a SF phase for smaller $t$ values.

Before we move on to the next section, we remark that by minimizing these contributions with respect to $\theta_{R}$ and $\theta_{E}$, we obtain $\tan \theta_{R}=\frac{\sqrt{2} \gamma_{R} \bar{\Delta}_{\uparrow} \bar{\Delta}_{\downarrow}}{t\left(\bar{\Delta}_{\uparrow}^{2}+\bar{\Delta}_{\downarrow}^{2}\right)}$ for the Rashba SOC and $\tan \left|\theta_{E}\right|=$ $\frac{2 \gamma_{E} \bar{\Delta}_{\uparrow} \bar{\Delta}_{\downarrow}}{t\left(\bar{\Delta}_{\uparrow}^{2}+\bar{\Delta}_{\downarrow}^{2}\right)}$ for the ERD SOC, respectively. In the simplest case when $h_{z}=0$, setting $\bar{\Delta}_{\uparrow}=\bar{\Delta}_{\downarrow}$ leads to $\tan \theta_{R}=\gamma_{R} /(t \sqrt{2})$ for the Rashba SOC and $\tan \left|\theta_{E}\right|=\gamma_{E} / t$ for the ERD SOC, which are in good agreement with our numerical results. In particular, we checked that $\tan \left|\theta_{E}\right|=2 \tan \theta_{R}$ is satisfied in Figs. 3(a) and 3(d) for any given $\mu$ and $t$ as long as both ground states are SF. Next, we are ready to analyze the ground-state phase diagrams in the presence of a general Zeeman field.

\section{MI-SF phase transitions: General Zeeman field}

So far, we argued that when $h_{y}=0$, while the MI-SF phase-transition boundaries are essentially identical for $\mathrm{ERD}_{x}$

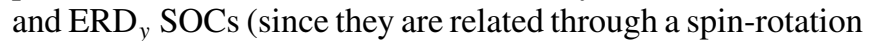
symmetry), their nonuniform-SF phases may be characterized by gauge-dependent order parameters. However, $h_{y} \neq 0$ breaks the symmetry between $\mathrm{ERD}_{x}$ and ERD $\mathrm{ESOCs}_{y}$ and, therefore, it is expected that $h_{y} \neq 0$ has dramatic consequences on the ground-state phase diagrams.

In Figs. 4-6, we show the $h_{y} \neq 0$ phase diagrams as functions of $\mu$ and $t$ for $\mathrm{ERD}_{x}, \mathrm{ERD}_{y}$, and Rashba SOCs, respectively. To understand the differences and similarities between these diagrams, first of all, we recall that when $h_{y}=0$ and $h_{z} \neq 0$, the symmetry between $\uparrow$ and $\downarrow$ bosons is broken and, therefore, they do not simultaneously become SF, unless the SOC strength is sufficiently strong. In a somewhat similar fashion, we find that increasing $h_{y}$ from 0 eventually causes the simultaneous transition of $\uparrow$ and $\downarrow$ bosons to SF, even with a relatively small $h_{y}=0.01 U$ for our chosen model parameters. This is because both the SOC and in-plane Zeeman field do not conserve spin, and they couple $\uparrow$ and $\downarrow$ bosons. However, the effects of on-site spin mixing due to $h_{y}$ are much stronger than that of the off-site spin mixing due to SOC. In addition, we note in all Figs. 4-6 that the sizes of the MI lobes shrink as a function of increasing SOC strength, which is a result of increased mobility of $\alpha$ particles, as mentioned in the previous section. However, the relative sizes of the MI lobes vary significantly depending on the symmetry of the SOC, and these differences can be understood from Eq. (14) as follows.

In Sec. III A, we argued that when there is no SOC, $\theta_{0 \downarrow}=\pi / 2$ as long as $h_{y} \neq 0$. Next, we show that an $\mathrm{ERD}_{x}$ SOC is not in competition with $h_{y}$ for the value of $\theta_{0 \downarrow}$, and this relation still holds. As noted in the previous section, $\theta_{y}=0$ for $\mathrm{ERD}_{x} \mathrm{SOC}$, and we find that $\theta_{0 \downarrow}=\pi / 2$ together with $\theta_{x} \in[-\pi / 2,0)$ minimize the contribution of the $\mathrm{ERD}_{x} \mathrm{SOC}$ to $E_{0}$. Note that this condition is not in conflict with the tunneling term since $\cos (x)$ is an even function. Minimizing the combined contributions of the tunneling and ERD $\mathrm{SOC}_{x}$ $-4 t\left(\bar{\Delta}_{\uparrow}^{2}+\bar{\Delta}_{\downarrow}^{2}\right)\left(\cos \theta_{x}+1\right)+8 \bar{\Delta}_{\uparrow} \bar{\Delta}_{\downarrow} \gamma_{x} \sin \theta_{x}$, with respect to $\theta_{x}$, we obtain $\tan \theta_{x}=-\frac{2 \gamma_{x} \bar{\Delta}_{\uparrow} \bar{\Delta}_{\downarrow}}{t\left(\bar{\Delta}_{\uparrow}^{2}+\bar{\Delta}_{\downarrow}^{2}\right)}$, which is similar to the expression we find in Sec. III B. In the simplest case when $h_{z}=0$, setting $\bar{\Delta}_{\uparrow}=\bar{\Delta}_{\downarrow}$ leads to $\tan \theta_{x}=-\gamma_{x} / t$. Thus, in the case of $\mathrm{ERD}_{x} \mathrm{SOC} h_{y} \neq 0$ clearly lifts the twofold degeneracy of $\theta_{0 \downarrow}= \pm \pi / 2$ solutions discussed in Sec. III B.

On the other hand, $\mathrm{ERD}_{y} \mathrm{SOC}$ is in competition with $h_{y}$ for the value of $\theta_{0 \downarrow}$, which can be easily inferred by looking at the two extreme limits. When $h_{y} \gg \gamma_{y} \rightarrow 0$, we already show in Sec. III A that $\theta_{0 \downarrow}=\pi / 2$. However, when $\gamma_{y} \gg h_{y} \rightarrow 0$, we show in Sec. III B that the ground state is twofold degenerate: both $\theta_{0 \downarrow}=0$ and $\pi$ minimize $E_{0}$. Thus, $\theta_{0 \downarrow}$ clearly depends on the ratio of $\gamma_{y}$ and $h_{y}$, and we may write $\theta_{0 \downarrow}=\pi / 2 \pm \eta$, where $\eta$ is determined by $\gamma_{y} / h_{y}$. Setting $\theta_{x}=0$ for the ERD $\mathrm{ESOC}_{y}$, and minimizing the combined contribution of tunneling and ERD $\mathrm{SOC}_{y} \mathrm{SOrms}$, $-4 t\left(\bar{\Delta}_{\uparrow}^{2}+\bar{\Delta}_{\downarrow}^{2}\right)\left(1+\cos \theta_{y}\right)-8 \bar{\Delta}_{\uparrow} \bar{\Delta}_{\downarrow} \gamma_{y} \cos \theta_{0 \downarrow} \sin \left|\theta_{y}\right|$, with respect to $\theta_{y}$, we obtain $\tan \left|\theta_{y}\right|=\frac{2 \gamma_{y} \cos \theta_{0 \downarrow} \bar{\Delta}_{\uparrow} \bar{\Delta}_{\downarrow}}{t\left(\bar{\Delta}_{\uparrow}^{2}+\bar{\Delta}_{\downarrow}^{2}\right)}$. In the simplest case when $h_{z}=0$, setting $\bar{\Delta}_{\uparrow}=\bar{\Delta}_{\downarrow}$ leads to tan $\left|\theta_{y}\right|=$ $\gamma_{y} \cos \theta_{0 \downarrow} / t$. In sharp contrast to the $\mathrm{ERD}_{x}$ SOC phase diagrams where a relatively small $h_{y}=0.01 U$ has sizable effects on the MI lobes as shown in Figs. 4(a)-4(c), it has a negligible effect on the ERD $\mathrm{SOC}_{y}$ diagrams that are shown in Figs. 5(a)-5(c). However, when the Zeeman field is sufficiently strong such as $h_{y}=0.1 U$, we see in Fig. 5 that ERD SOC $^{2}$ has negligible effects.

These findings may explain why the effects of $h_{y} \neq 0$ on the ground-state phase diagrams of Rashba SOC are stronger (weaker) than those of $\mathrm{ERD}_{y}\left(\mathrm{ERD}_{x}\right) \mathrm{SOC}$. This is because while the $h_{y}$ term competes with the $\gamma_{y}$ component of the Rashba SOC for the value of $\theta_{0 \downarrow}$, it does not compete with the $\gamma_{x}$ component. Therefore, $h_{y} \neq 0$ has an intermediate effect on the phase diagrams of the Rashba SOC. Similar to the ERD SOC, it should not be surprising that the Rashba SOC is also competing with the $h_{y}$ term for the value of $\theta_{0 \downarrow}$, which can 



FIG. 4. (Color online) Ground-state phase diagrams with $\operatorname{ERD}_{x} \operatorname{SOCs}\left(\gamma_{x}=\gamma_{E}, \gamma_{y}=0\right)$ and general Zeeman fields. The rest of the parameters are specified in Fig. 3.

again be easily inferred by looking at the two extreme limits. When $h_{y} \gg \gamma_{R} \rightarrow 0$, we already show in Sec. III A that $\theta_{0 \downarrow}=$ $\pi / 2$. However, when $\gamma_{R} \gg h_{y} \rightarrow 0$, we show in Sec. III B that the ground state is fourfold degenerate: both $\theta_{0 \downarrow}= \pm \pi / 4$ and $\pm 3 \pi / 4$ minimize $E_{0}$. Thus, $\theta_{0 \downarrow}$ clearly depends on the ratio of $\gamma_{R}$ and $h_{y}$, and we may write $\theta_{0 \downarrow}=\pi / 2 \pm \eta$, where $\eta \in(0, \pi / 4)$ is determined by $\gamma_{R} / h_{y}$. This discussion shows that $h_{y} \neq 0$ reduces the fourfold degeneracy of $\theta_{0 \downarrow}$ to twofold. In addition, we also note that $h_{y} \neq 0$ breaks the rotational $x y$ symmetry and, therefore, $\left|\theta_{x}\right|$ and $\left|\theta_{y}\right|$ are not necessarily equal to each other. This completes our analysis of in-plane Zeeman field on the ground-state phase diagrams, and we are ready to
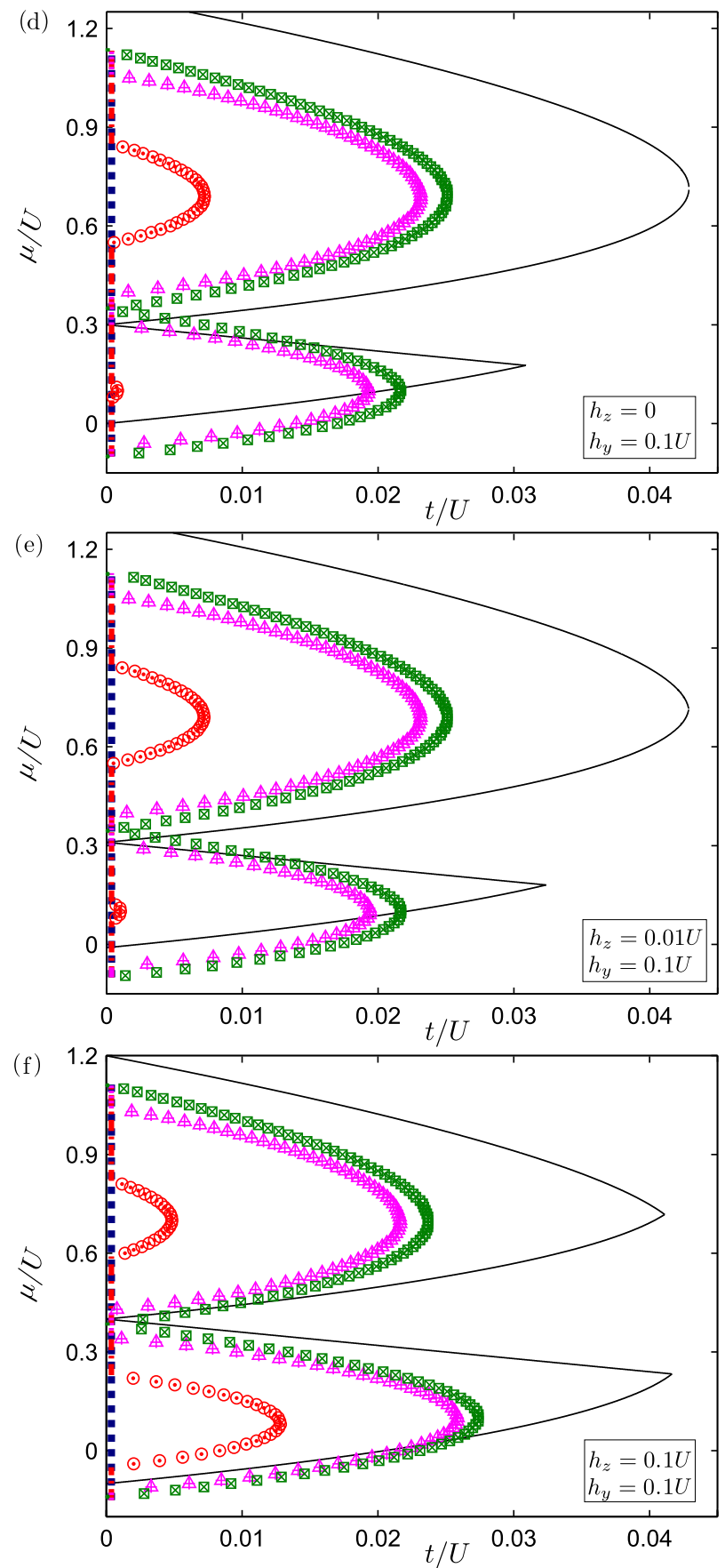

conclude the paper with a brief summary of our results and an outlook.

\section{SUMMARY AND OUTLOOK}

To conclude, here we considered a square lattice in two dimensions and studied the effects of both the strength and symmetry of SOC and Zeeman field on the ground-state phases and phase diagram of the two-component Bose-Hubbard model. In particular, based on a variational Gutzwiller ansatz, we analyzed the competition between the interaction, tunneling, Rashba and ERD SOCs, and out-of- and in-plane Zeeman 

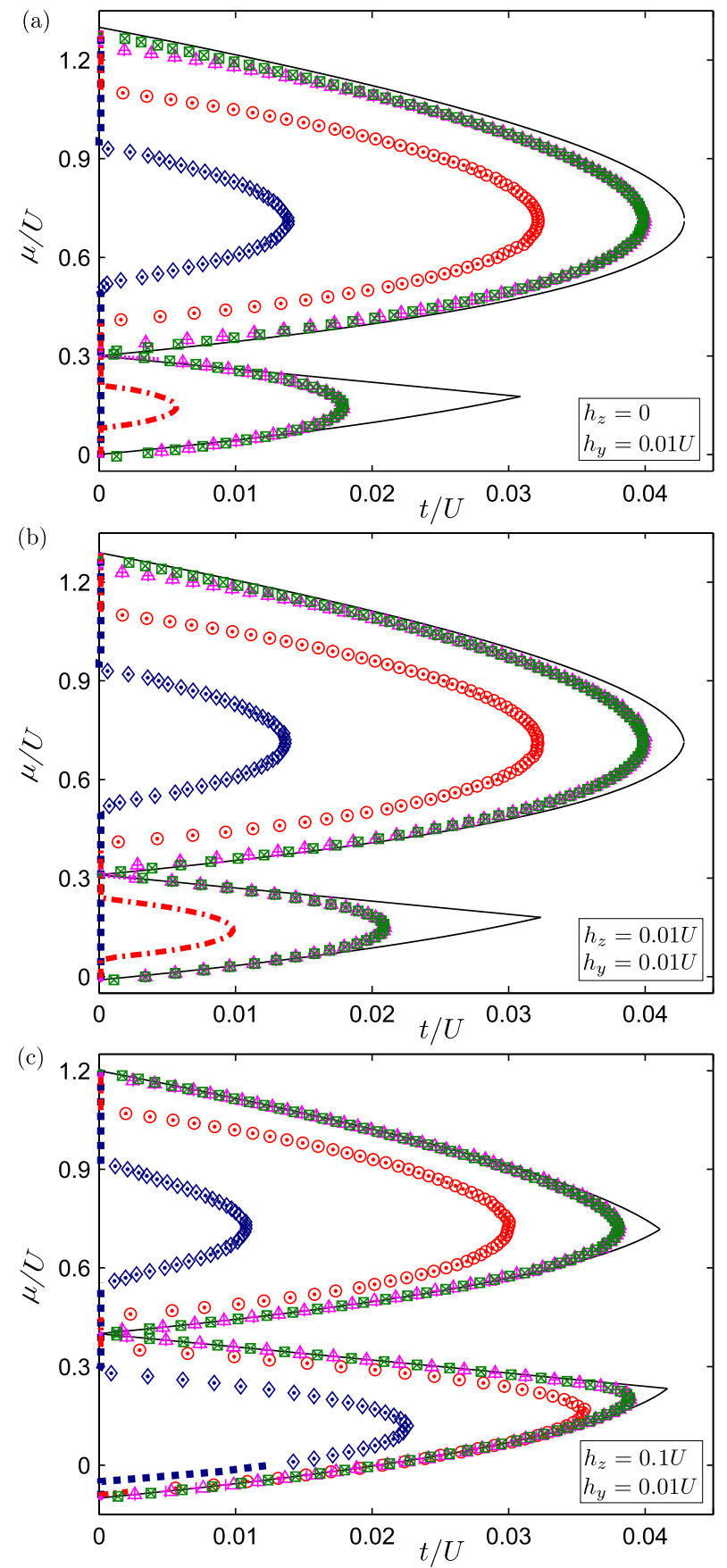

FIG. 5. (Color online) Ground-state phase diagrams with ERD $\operatorname{SOCs}\left(\gamma_{x}=0, \gamma_{y}=\gamma_{E}\right)$ and general Zeeman fields. The rest of the parameters are specified in Fig. 3.

fields on the MI-SF phase-transition boundary and the nature of the SF phase nearby. It is already established in the literature that this method is equivalent to the mean-field decoupling theory, at least in the absence of a SOC, and, therefore, it is expected to give a qualitatively accurate description of the MI and SF phases. In addition to the phase diagrams, one of our main results is as follows: Gutzwiller calculations showed that while the magnitudes of the order parameters are uniform across the entire lattice, their phases may vary from site to site due to SOC, and, therefore, the SF phase is a nonuniform one. We gave a complete account and intuitive understanding of this SOC-induced nonuniform-SF phase and its resultant
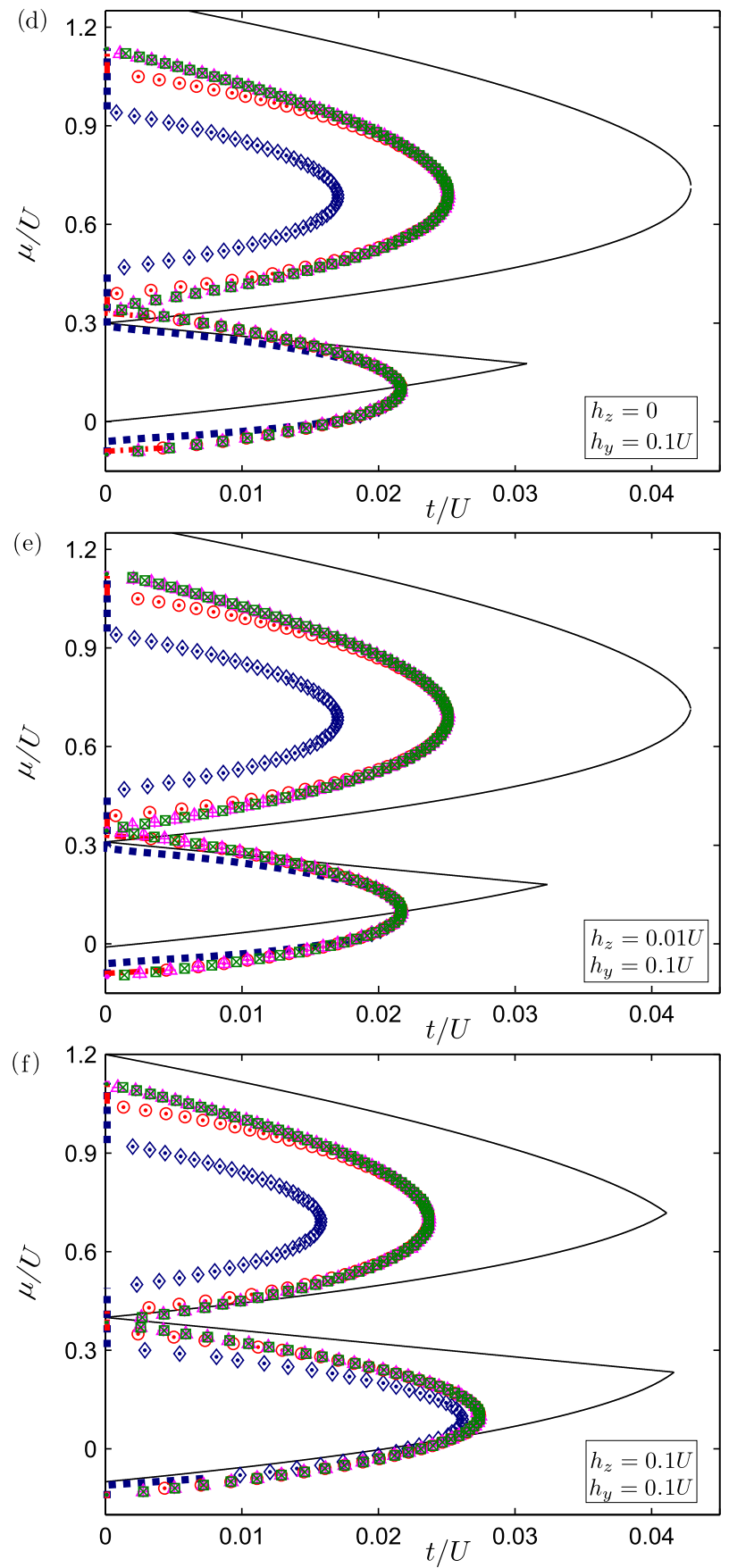

phase patterns by supporting our numerical calculations with fully analytical insights.

One may extend this work in many directions. For instance, as we emphasized in the main text, recently proposed exotic magnetic phases exhibiting spin textures in the form of spin spirals and vortex and Skyrmion crystals inside the MI lobes [36-40] are not accessible within a variational Gutzwiller ansatz. However, there is no a priori reason why the method cannot be used to investigate the spin-textured nonuniform-SF phases. Since spin-textured SF phases have only been discussed in the literature for a weakly interacting Bose gas, such studies are especially of interest near the MI-SF 

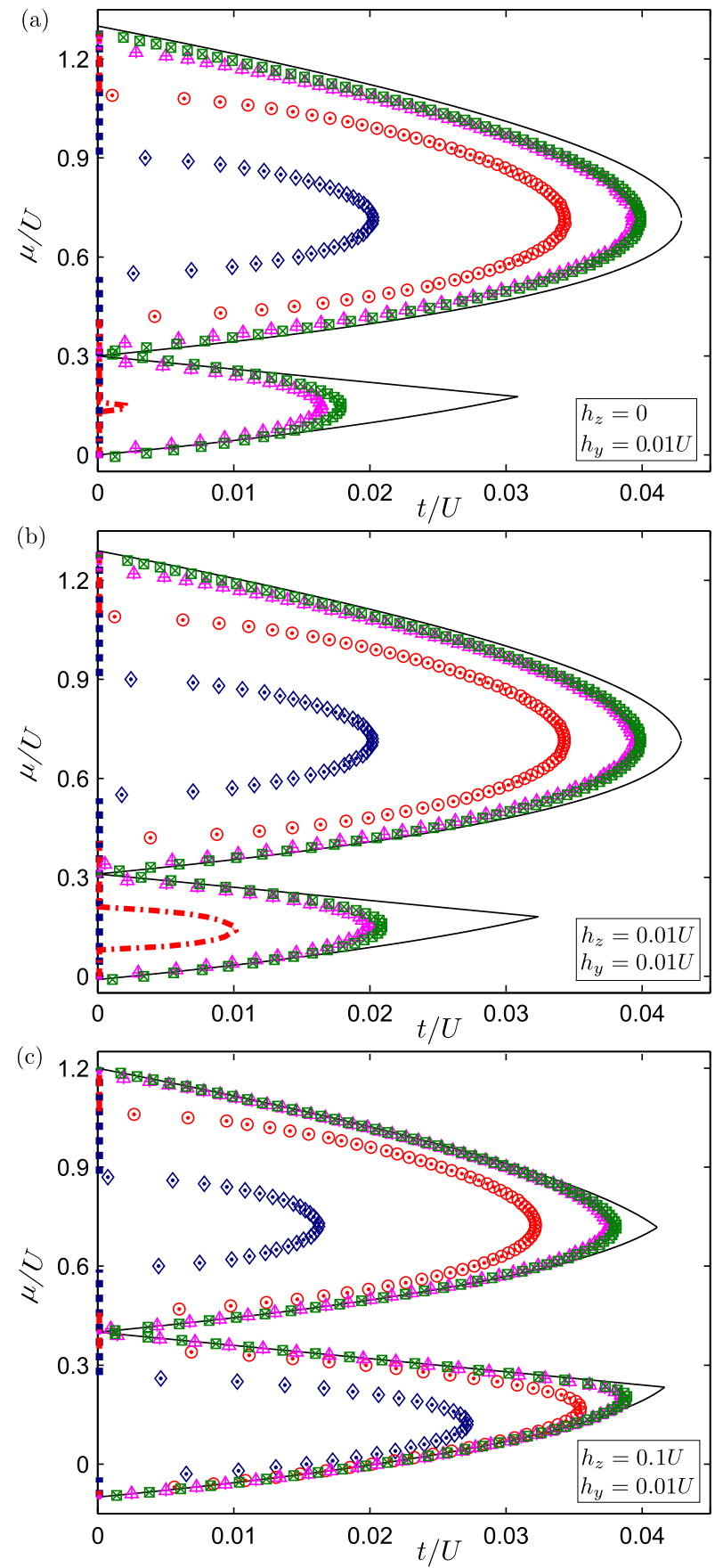
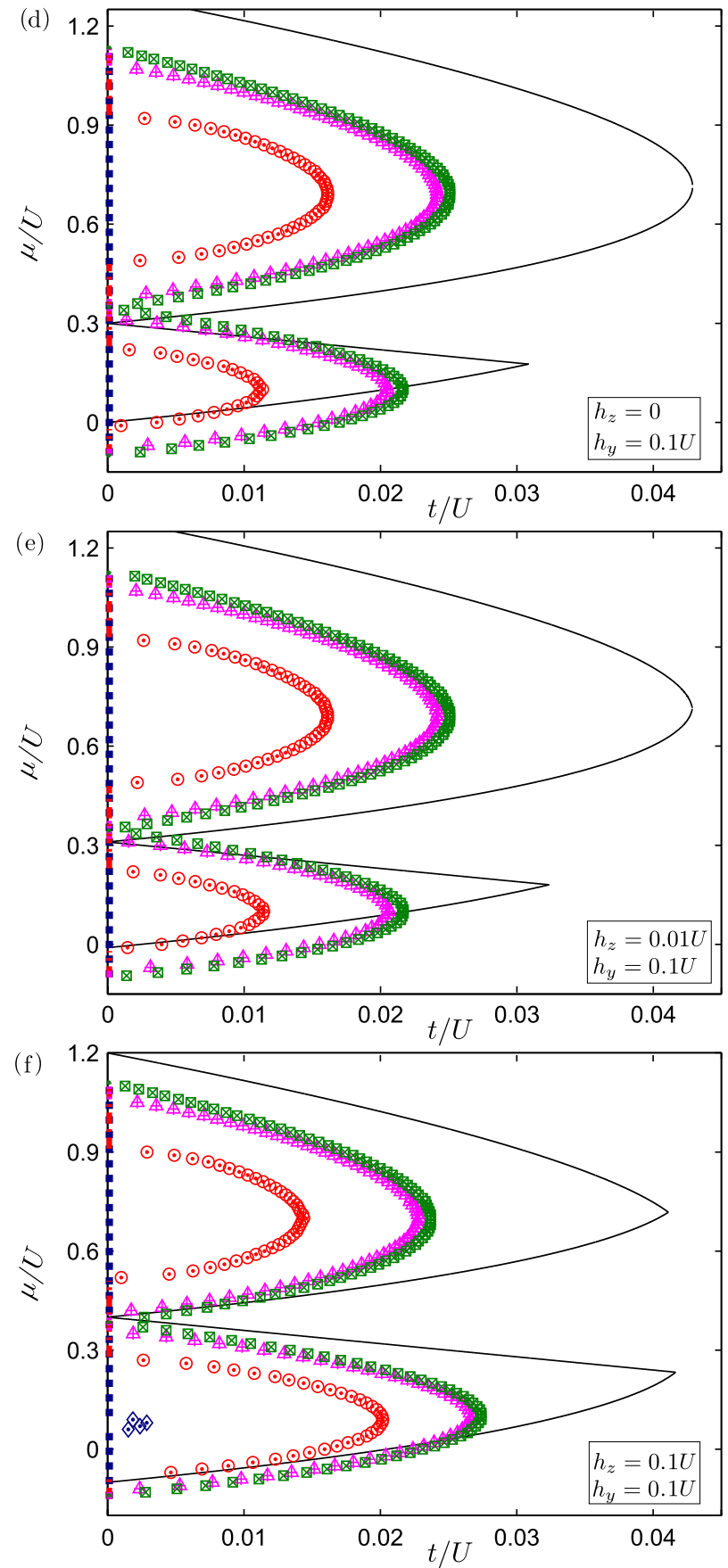

FIG. 6. (Color online) Ground-state phase diagrams with Rashba SOCs $\left(\gamma_{x}=\gamma_{y}=\gamma_{R}\right)$ and general Zeeman fields. The rest of the parameters are specified in Fig. 3.

phase-transition boundary and the strongly interacting regime. In addition, SF phases in this work are characterized by the order parameter $\Delta_{j \alpha}=\left\langle\hat{a}_{j \alpha}\right\rangle$, and, therefore, single-particle and/or single-hole excitations are always gapped inside our MI lobes. However, this definition of the SF phase does not discriminate the possibility of exotic multiparticle and/or multihole excitations that may be gapless; our MI lobes may still have some sort of hidden SF orders. For instance, in the absence of SOC and Zeeman field, it is already known that a counterflow-SF phase of particle-hole pairs [24,25,29,31] characterized by the order parameter $\Delta_{j} \equiv\left\langle\hat{a}_{j \uparrow} \hat{a}_{j \downarrow}^{\dagger}\right\rangle$ and a paired SF phase of two particles or two holes [25,28-31] characterized by the order parameter $\Delta_{j} \equiv\left\langle\hat{a}_{j \uparrow} \hat{a}_{j \downarrow}\right\rangle$ are possible when $U_{\uparrow \downarrow}>0$ and $U_{\uparrow \downarrow}<0$, respectively [31]. The effects of SOC and/or Zeeman field on the fates of such exotic SF phases is uncharted territory.

\section{ACKNOWLEDGMENTS}

A.T.B. is supported by the TÜBITAK 2218 Domestic Postdoctoral Fellowship Program, and M.I. is supported by the Marie Curie IRG Grant No. FP7-PEOPLE-IRG-2010268239, TÜBITAK Career Grant No. 3501-110T839, and TÜBA-GEBIP. 
[1] M. Lewenstein, A. Sanpera, V. Ahufinger, B. Damski, A. Sen De, and U. Sen, Adv. Phy. 56, 243 (2007).

[2] I. Bloch, J. Dalibard, and W. Zwerger, Rev. Mod. Phys. 80, 885 (2008).

[3] S. Giorgini, L. P. Pitaevskii, and S. Stringari, Rev. Mod. Phys. 80, 1215 (2008).

[4] M. P. A. Fisher, P. B. Weichman, G. Grinstein, and D. S. Fisher, Phys. Rev. B 40, 546 (1989).

[5] M. Z. Hasan and C. L. Kane, Rev. Mod. Phys. 82, 3045 (2010).

[6] X.-L. Qi. and S.-C. Zhang, Rev. Mod. Phys. 83, 1057 (2011).

[7] J. Maciejko, T. L. Hughes, and S.-C. Zhang, Annu. Rev. Cond. Mater. Phys. 2, 31 (2011).

[8] J. Dalibard, F. Gerbier, G. Juzelinas, and P. Öhberg, Rev. Mod. Phys. 83, 1523 (2011).

[9] V. Galitski and I. B. Spielman, Nature (London) 494, 49 (2013).

[10] T. D. Stanescu, B. Anderson, and V. Galitski, Phys. Rev. A 78, 023616 (2008).

[11] Y.-J. Lin, Y.-J. Lin, K. Jiménez-García, and I. B. Spielman, Nature (London) 471, 83 (2011).

[12] J.-Y. Zhang, S.-C. Ji, Z. Chen, L. Zhang, Z.-D. Du, B. Yan, G.-S. Pan, B. Zhao, Y.-J. Deng, H. Zhai, S. Chen, and J.-W. Pan, Phys. Rev. Lett. 109, 115301 (2012).

[13] P. Wang, Z.-Q. Yu, Z. Fu, J. Miao, L. Huang, S. Chai, H. Zhai, and J. Zhang, Phys. Rev. Lett. 109, 095301 (2012).

[14] L. W. Cheuk, A. T. Sommer, Z. Hadzibabic, T. Yefsah, W. S. Bakr, and M. W. Zwierlein, Phys. Rev. Lett. 109, 095302 (2012).

[15] C. Qu, C. Hamner, M. Gong, C. Zhang, and P. Engels, Phys. Rev. A 88, 021604(R) (2013).

[16] Z. Fu, L. Huang, Z. Meng, P. Wang, X.-J. Liu, H. Pu, H. Hu, and J. Zhang, Phys. Rev. A 87, 053619 (2013).

[17] R. A. Williams, M. C. Beeler, L. J. LeBlanc, K. Jiménez-García, and I. B. Spielman, Phys. Rev. Lett. 111, 095301 (2013).

[18] C. J. Kennedy, G. A. Siviloglou, H. Miyake, W. C. Burton, and W. Ketterle, Phys. Rev. Lett. 111, 225301 (2013).

[19] K. Sheshadri, H. R. Krishnamurthy, R. Pandit, and T. V. Ramakrishnan, Europhys. Lett. 22, 257 (1993).

[20] D. van Oosten, P. van der Straten, and H. T. C. Stoof, Phys. Rev. A 63, 053601 (2001).

[21] J. K. Freericks and H. Monien, Phys. Rev. B 53, 2691 (1996).

[22] J. K. Freericks, H. R. Krishnamurthy, Y. Kato, N. Kawashima, and N. Trivedi, Phys. Rev. A 79, 053631 (2009).

[23] L. Pollet, arXiv:1307.5430.

[24] A. B. Kuklov and B. V. Svistunov, Phys. Rev. Lett. 90, 100401 (2003).
[25] E. Altman, W. Hofstetter, E. Demler, and M. D. Lukin, New J. Phys. 5, 113 (2003).

[26] A. Kuklov, N. Prokof'ev, and B. Svistunov, Phys. Rev. Lett. 92, 050402 (2004).

[27] A. Isacsson, M.-C. Cha, K. Sengupta, and S. M. Girvin, Phys. Rev. B 72, 184507 (2005).

[28] A. Argüelles and L. Santos, Phys. Rev. A 75, 053613 (2007).

[29] Anzi Hu, L. Mathey, I. Danshita, E. Tiesinga, C. J. Williams, and C. W. Clark, Phys. Rev. A 80, 023619 (2009).

[30] M. Iskin, Phys. Rev. A 82, 033630 (2010).

[31] T. Ozaki, I. Danshita, and T. Nikuni, arXiv:1210.1370.

[32] G. Thalhammer, G. Barontini, L. De Sarlo, J. Catani, F. Minardi, and M. Inguscio, Phys. Rev. Lett. 100, 210402 (2008).

[33] A. Widera, S. Trotzky, P. Cheinet, S. Fölling, F. Gerbier, I. Bloch, V. Gritsev, M. D. Lukin, and E. Demler, Phys. Rev. Lett. 100, 140401 (2008).

[34] D. M. Weld, P. Medley, H. Miyake, D. Hucul, D. E. Pritchard, and W. Ketterle, Phys. Rev. Lett. 103, 245301 (2009).

[35] B. Gadway, D. Pertot, R. Reimann, and D. Schneble, Phys. Rev. Lett. 105, 045303 (2010).

[36] W. S. Cole, S. Zhang, A. Paramekanti, and N. Trivedi, Phys. Rev. Lett. 109, 085302 (2012).

[37] J. Radić, A. Di Ciolo, K. Sun, and V. Galitski, Phys. Rev. Lett. 109, 085303 (2012).

[38] Z. Cai, X. Zhou, and C. Wu, Phys. Rev. A 85, 061605(R) (2012).

[39] X. Zhou, Y. Li, Z. Cai, and C. Wu, J. Phys. B: At. Mol. Opt. Phys. 46, 134001 (2013).

[40] D.-W. Zhang, J.-P. Chen, C.-J. Shan, Z. D. Wang, and S.-L. Zhu, Phys. Rev. A 88, 013612 (2013).

[41] J. Zhao, S. Hu, J. Chang, P. Zhang, and X. Wang, arXiv:1308.6710.

[42] S. Mandal, K. Saha, and K. Sengupta, Phys. Rev. B 86, 155101 (2012).

[43] A. Dutta and S. Mandal, Phys. Rev A 88, 063619 (2013).

[44] T. Grass, K. Saha, K. Sengupta, and M. Lewenstein, Phys. Rev. A 84, 053632 (2011).

[45] C. H. Wong and R. A. Duine, Phys. Rev. Lett. 110, 115301 (2013); Phys. Rev. A 88, 053631 (2013).

[46] T. Grass, B. Juliá-Díaz, M. Burrello, and M. Lewenstein, J. Phys. B: At. Mol. Opt. Phys. 46, 134006 (2013).

[47] H. Sakaguchi and Ben Li, Phys. Rev. A 87, 015602 (2013).

[48] C. Wu, I. Mondragon-Shem, and X.-F. Zhou, Chin. Phys. Lett. 28, 097102 (2011). 